\title{
MicroRNA-100 functions as a tumor suppressor by inhibiting Lgr5 expression in colon cancer cells
}

\author{
MING-KAI ZHOU ${ }^{1}$, XIAO-JUN LIU ${ }^{1}$, ZHI-GUO ZHAO ${ }^{2}$ and YI-MENG CHENG ${ }^{3}$ \\ ${ }^{1}$ Intensive Care Unit and ${ }^{2}$ Department of Gastroenterology, The Second Affiliated Hospital of Zhengzhou University, \\ Zhengzhou, Henan 450014; ${ }^{3}$ Department of Neurology, The Third Hospital of Zhengzhou, \\ Zhengzhou, Henan 450000, P.R. China
}

Received February 11, 2014; Accepted October 1, 2014

DOI: $10.3892 / \mathrm{mmr} .2014 .3052$

\begin{abstract}
Previous studies have demonstrated that microRNAs (miRNAs), a class of single-stranded RNA molecules that are 18-27 nucleotides in length, serve a critical function in tumorigenesis, including in the development of colon cancer. In the current study, miR-100 levels were demonstrated to be reduced in colon cancer tissues compared with the levels in matched adjacent normal tissues. Forced overexpression of miR-100 by transfection with miR-100 mimics substantially inhibited the proliferation, migration and invasion of SW480 and HCT116 cells, whereas reduced expression, resulting from transfection of antisense oligonucleotides, promoted these processes. At the molecular level, miR-100 was observed to reduce the levels of leucine-rich repeat-containing $\mathrm{G}$ protein-coupled receptor 5 ( $\operatorname{Lgr} 5)$, by binding to its 3 -untranslated region. As a result of this, Wnt/ $\beta$-catenin signaling was affected by fluctuations in the level of miR-100 mimics or antisense. Collectively, the results of the current study elucidate a novel regulatory pathway involving miR-100 and Lgr5 in colon cancer cells, which may present a potential therapeutic target.
\end{abstract}

\section{Introduction}

Colon cancer is the third most common type of cancer and the leading cause of cancer-associated mortality in Western communities $(1,2)$. In China, the incidence is lower than in Western countries, but nevertheless is a substantial burden (3). The molecular mechanisms underlying the development of this type of cancer remain to be fully elucidated, thus there currently exist limited therapeutic options (4).

Correspondence to: Dr Xiao-Jun Liu, Intensive Care Unit, The Second Affiliated Hospital of Zhengzhou University, No. 2 Jingba Road, Zhengzhou, Henan 450014, P.R. China

E-mail: hnxiaojunliu@126.com

Key words: colon cancer, microRNA, microRNA-100, $\mathrm{G}$ protein-coupled receptor $5, \beta$-catenin
MicroRNAs (miRNAs) are a family of small non-coding RNA molecules of 18-27 nucleotides in length (5). In general, miRNAs negatively regulate gene expression by binding to the 3'-untranslated region (3'-UTR) of their target double-stranded mRNA, leading to degradation of the mRNA via the Dicer complex (6). The abnormal expression of certain miRNAs has been observed in various types of solid tumor, including colon cancer (7). For example, miRNA-21 has recently emerged as a novel biomarker in colon cancer, with the potential to be used as a diagnostic and therapeutic target (8). Additionally, several miRNAs have been reported to regulate colon cell growth, migration and invasion, including miR-32, -224 and -203 (9-11).

The current study investigated miR-100 using gain- and loss-of-function experiments, and the effect of up- or downregulation of miR-100 on the proliferation, migration and invasion of colon cells was determined.

\section{Materials and methods}

Human tissues. A total of 25 pairs of frozen primary colon cancer samples and corresponding histologically normal mucosa samples were obtained from the Department of Gastroeterology, Second Affiliated Hospital of Zhengzhou University (Zhengzhou, China). The diagnoses of these tissue samples were verified by pathologists. The current study was approved by the Ethics Committee of the Second Affiliated Hospital of Zhengzhou University.

Cell culture. The SW480 and HCT116 colon cancer cell lines were purchased from the cell bank of the Type Culture Collection of The Chinese Academy of Sciences (Shanghai, China), and cultured in Dulbecco's modified Eagle's medium (DMEM) supplemented with $10 \%$ fetal bovine serum, $100 \mathrm{IU} / \mathrm{ml}$ penicillin and $100 \mathrm{mg} / \mathrm{ml}$ streptomycin (all from Gibco Life Technologies, Carlsbad, CA, USA).

Cell transfection. miR-100 mimics, antisense oligonucleotides and negative controls (NCs) were purchased from Shanghai Genepharma Co., Ltd. (Shanghai, China). Transfections were performed using Lipofectamine 2000 (Invitrogen Life Technologies, Carlsbad, CA, USA), according to the manufacturer's instructions. 
RNA isolation and quantitative polymerase chain reaction $(q P C R)$. RNA was isolated from cells using TRIzol reagent (Invitrogen Life Technologies), and reverse transcription was performed with the Takara RNA PCR kit (Takara Biotechnology Co., Ltd., Dalian, China), according to the manufacturer's instructions. In order to determine the transcripts of the genes of interest, qPCR was performed using a SYBR Premix Ex Taq master mix (Takara Biotechnology Co., Ltd.) with an ABI 7500 Real-Time PCR system (Applied Biosystems Life Technologies, Foster City, CA, USA). PCR cycling conditions included an initial holding period at $94^{\circ} \mathrm{C}$ for $5 \mathrm{~min}$, followed by a two-step PCR program consisting of 40 cycles of $94^{\circ} \mathrm{C}$ for $5 \mathrm{sec}$ and $60^{\circ} \mathrm{C}$ for $30 \mathrm{sec}$. Expression of U6 small nuclear RNA was determined as an internal control. Primer sequences were as follows: Cyclin D1, F 5'-GCTGCG AAGTGGAAACCATC-3' and R 5'-CCTCCTTCTGCA CACATTTGAA-3'; cyclin E, F 5'-AAGGAGCGGGACACC ATGA-3' and R 5'-ACGGTCACGTTTGCCTTCC-3'.

Bromodeoxyuridine (BrdU) incorporation assays. For the BrdU incorporation assays, a BrdU cell proliferation enzyme-linked immunosorbent assay kit (Beyotime Institute of Biotechnology, Shanghai, China) was used to analyze the incorporation of BrdU during the $\mathrm{S}$ phase of the cell cycle in the SW480 and HCT116 cells, in accordance with the manufacturer's instructions. All experiments were repeated a minimum of three times in quadruplicate.

MTT assay. The cell viability was determined by assaying the reduction of 3-(4, 5-dimethylthiazol-2-yl)-2, 5-di-phenyltetrazolium bromide (MTT) to formazan. SW480 and HCT116 cells were cultured in DMEM at a concentration of $60-70 \%$, prior to the addition of $100 \mu \mathrm{l}$ MTT (Beyotime Institute of Biotechnology) for $4 \mathrm{~h}$. The cell viability in the different samples was measured using a Raman spectrophotometer (B\&W Tek, Inc., Newark, DE, USA) at a wavelength of $470 \mathrm{~nm}$.

Cell migration and invasion assays. Subsequent to transfection of the SW480 and HCT116 cells with miR-100 mimics, antisense or NC for $24 \mathrm{~h}$, cell migration and invasion were analyzed. The cells were seeded in Transwell migration or extracellular matrix-coated invasion chambers (Tumor Cell Transendothelial Migration Assay kit and ECMatrix Cell Invasion Assay kit, respectively; EMD Millipore, Temecula, CA, USA) and incubated for a further $24 \mathrm{~h}$. Subsequently, the Transwell migration assay or invasion assay was conducted according to manufacturer's instructions. Cell migration and invasion were quantified with an iMark Microplate Absorbance Reader (\#168-1130; Bio-Rad Laboratories, Hercules, CA, USA) at $570 \mathrm{~nm}$, according to the manufacturer's instructions.

Luciferase reporter assay. Total cDNA from SW480 cells was obtained from mRNA using a Reverse Transcription System (Promega Corporation, Madison, WI, USA) according to the manufacturer's protocols. The cDNA was used to amplify the 3'-UTR of Lgr5 by PCR. The Lgr5 3'UTR was cloned into pMir-Report (Ambion Life Technologies, Shanghai, China), yielding pMir-Report-Lgr5. Mutations were introduced

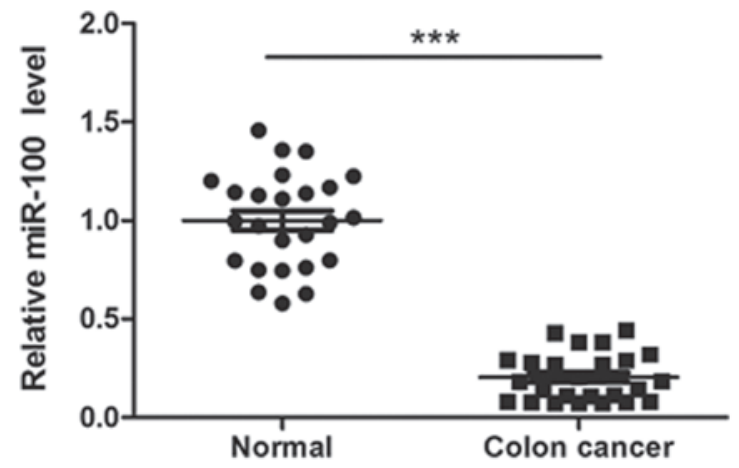

Figure 1. Expression levels of miR-100 in colon cancer tissues. The miR-100 expression levels were determined by RT-qPCR in human colon cancer tissues and adjacent normal tissues. ${ }^{* * *} \mathrm{P}<0.001$ vs. normal tissues. miR-100, microRNA-100; RT-qPCR, reverse transcription-quantitative polymerase chain reaction.

in potential miR-100 binding sites using the QuikChange site-directed mutagenesis kit (Agilent Technologies, Shanghai, China). Cells were transfected with the pMir-Report vectors containing the 3'-UTR variants with miR-100 mimics or antisense for $36 \mathrm{~h}$. The pRL-TK vector (Promega Corporation) carrying the Renilla luciferase gene was used as an internal control to normalize the transfection efficiency. Luciferase values were determined using the Dual-Luciferase Reporter Assay System (Promega Corporation). miRWalk software (www.umm.uni-heidelberg.de/apps/zmf/mirwalk/) was used for analysis.

Western blot. Following transfection of mimics, antisense or NC, tissues or SW480 cells were lysed with RIPA buffer (Beyotime Institute of Biotechnology). The protein (40 $\mu \mathrm{g}$ ) was subjected to $7.5 \%$ SDS-PAGE (Shanghai Sangong Pharmaceutical Co., Ltd., Shanghai, China) on a PowerPAC HC High-Current Power Supply electrophoresis machine (Bio-Rad Laboratories), and separated proteins were transferred to nitrocellulose membranes (EMD Millipore). The membranes were incubated overnight at $4^{\circ} \mathrm{C}$ with the following antibodies: Monoclonal mouse anti-human $\beta$-actin (1:1,000; sc-130065); polyclonal rabbit anti-human leucine-rich-repeat-containing $\mathrm{G}$ protein-coupled receptor 5 (Lgr5; 1:2,000; sc-135238); monoclonal rabbit anti-human $\beta$-catenin (1:2,000; sc-376841); and polyclonal goat anti-human histone H1 (1:1,000; sc-247158) (all Santa Cruz Biotechnology, Inc., Dallas, TX, USA). The immunoreactive bands were detected with a ChemiGlow West Chemiluminescence Substrate kit (ProteinSimple, Santa Clara, CA, USA) with the FluorChem FC2 system (NtureGene Corporation, Beijing, China).

Statistical analysis. Data are presented as the mean \pm standard error. Statistical analysis was performed with SPSS, version 13.0 (SPSS, Inc., Chicago, IL, USA). P<0.05 was considered to indicate a statistically significant difference.

\section{Results}

miR-100 is downregulated in colon cancer tissues. The expression level of miR-100 in 25 pairs of human tissues 
A

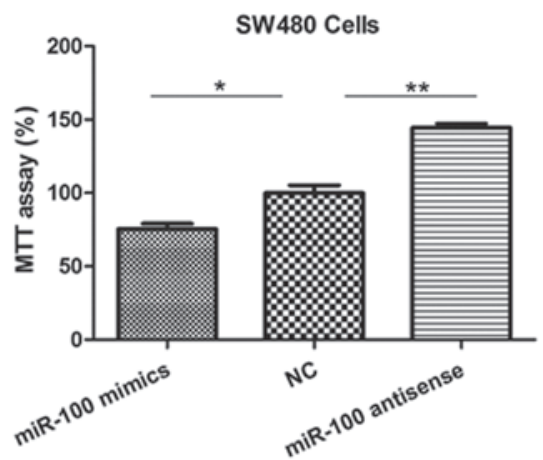

B

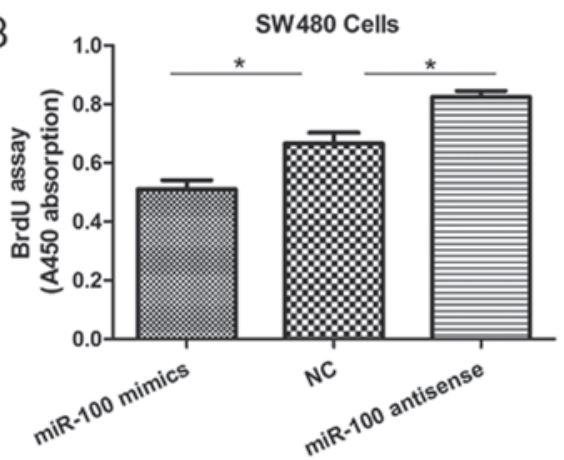

C

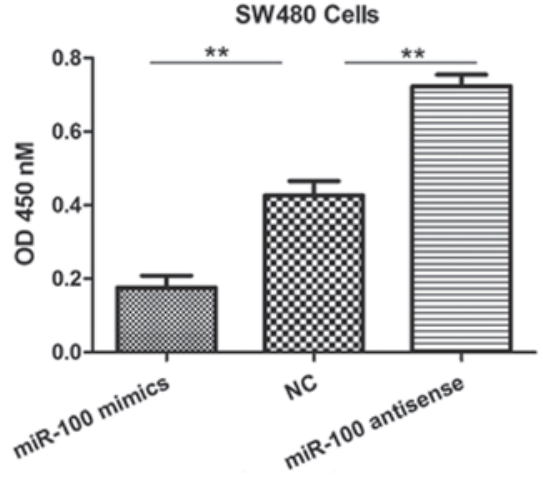

D

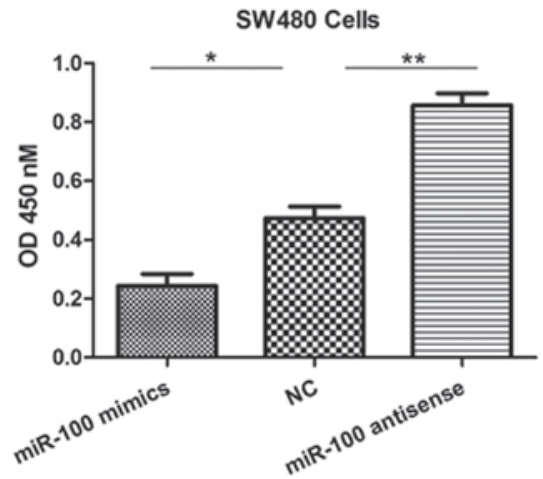

Figure 2. miR-100 mimics or antisense oligonucleotides regulate SW480 cell viability, proliferation, migration and invasion. (A) The MTT assay indicated a reduction or increase in cell viability following transfection of mimics or antisense, respectively. (B) The BrdU assay indicated a reduction or increase in proliferation following transfection of mimics or antisense oligonucleotides, respectively. (C) The Transwell migration assay indicated a reduction or increase in migration following transfection of mimics or antisense oligonucleotides, respectively. (D) The invasion assay indicated a reduction or increase in invasion capabilities following transfection of mimics or antisense oligonucleotides, respectively. ${ }^{*} \mathrm{P}<0.05$ and ${ }^{* * *} \mathrm{P}<0.01$ vs. NC. miR-100, microRNA-100; BrdU, bromodeoxyuridine; NC, negative control; OD, optical density.
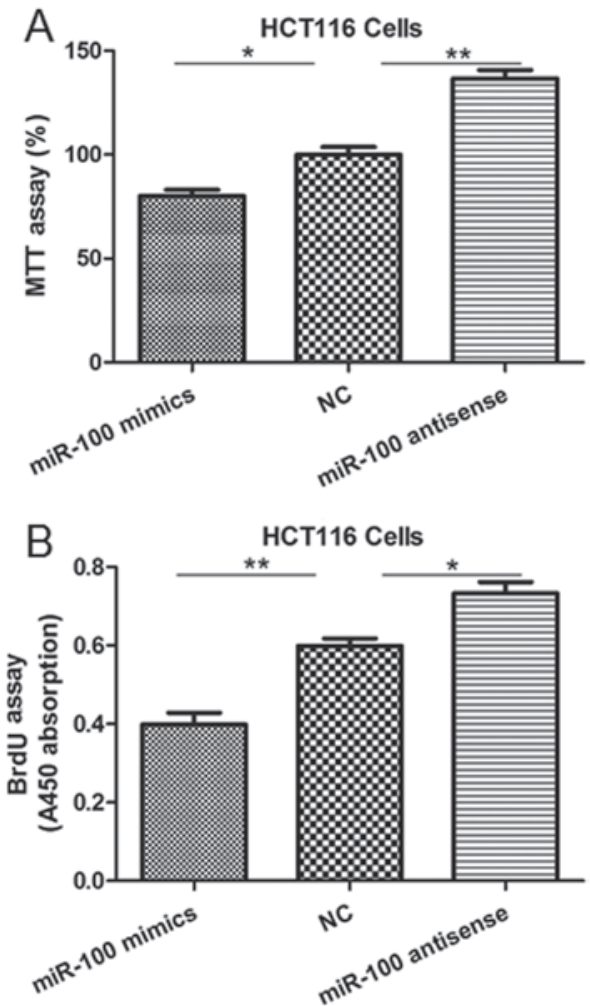

C HCT116 Cells
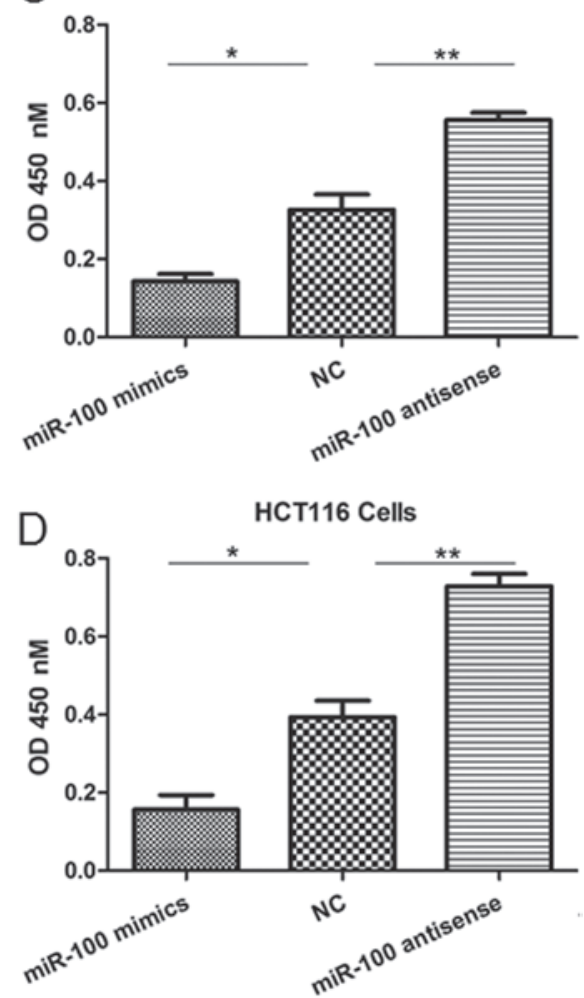

Figure 3. miR-100 mimics or antisense oligonucleotides regulate HCT116 cell viability, proliferation, migration and invasion. (A) The MTT assay indicated a reduction or increase in cell viability following transfection of mimics or antisense oligonucleotides, respectively. (B) The BrdU assay indicated a reduction or increase in proliferation following transfection of mimics or antisense oligonucleotides, respectively. (C) The Transwell migration assay indicated a reduction or increase in migration following transfection of mimics or antisense oligonucleotides, respectively. (D) The invasion assay indicated a reduction or increase in cell viability following transfection of mimics or antisense oligonucleotides, respectively. "P<0.05 and ${ }^{* * *} \mathrm{P}<0.01$ vs. NC. miRNA-100, microRNA-100; BrdU, bromodeoxyuridine; $\mathrm{NC}$, negative control; OD, optical density. 


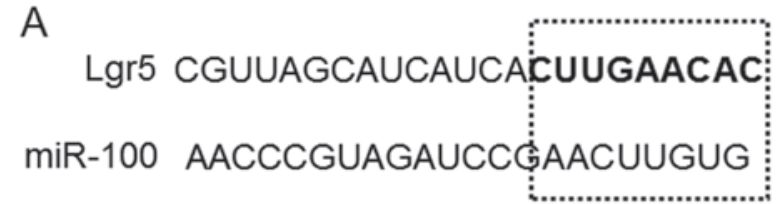

B

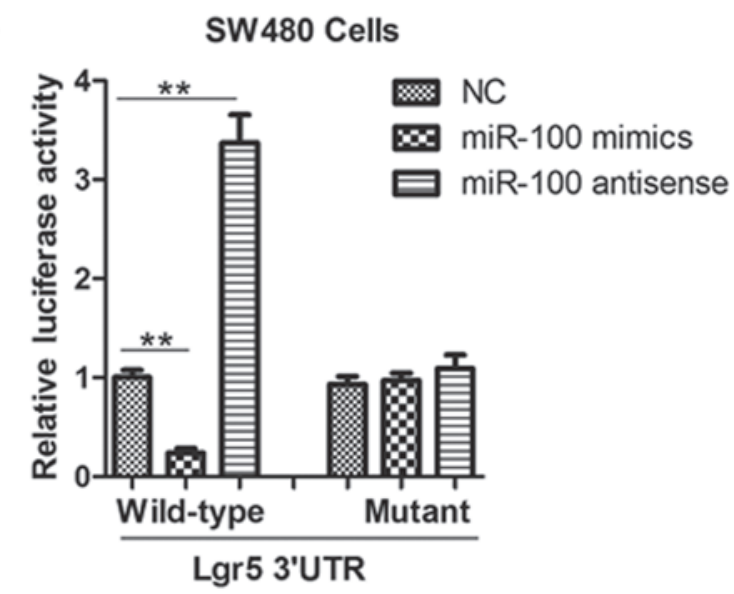

C

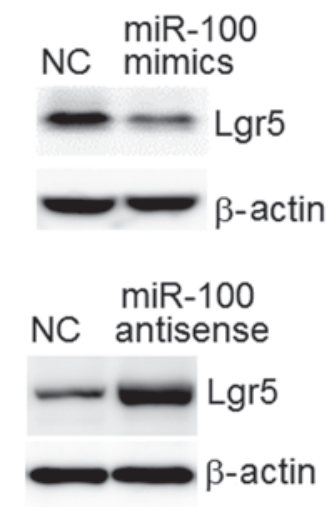

Figure 4. miR-100 downregulated the expression of Lgr5 protein by targeting its 3'-UTR. (A) Prediction of miR-100 binding sites in the 3'-UTRs of the human Lgr5 gene by miRWalk bioinformatics software. A potential binding site is highlighted in bold. (B) Luciferase reporter assays in SW480 cells. Cells were transfected with wild-type or mutant 3'-UTR-reporter constructs with miR-100 mimics or antisense ${ }^{* * *} \mathrm{P}<0.01$ vs. NC. Protein levels of Lgr5 were determined by western blot in SW480 cells transfected with miR-100 (C) mimics or (D) antisense. miR-100, microRNA-100; UTR, untranslated region; NC, negative control.

was measured by RT-qPCR. As demonstrated in Fig. 1, the miR-100 expression level was significantly lower in colon cancer tissues compared with the level in matched adjacent normal tissues $(\mathrm{P}<0.001)$.

miR-100 reduces the viability, proliferation, migration and invasion of colon cancer cells. To investigate the function of miR-100 in tumorigenesis, gain- and loss-of-function experiments involving the introduction of mimics or antisense oligonucleotides into SW480 and HCT116 cells were performed. Scramble sequences were used for the NC group. As observed with the MTT and BrdU incorporation assays, cell viability and proliferation levels were significantly reduced in SW480 cells in which miR-100 was overexpressed, but

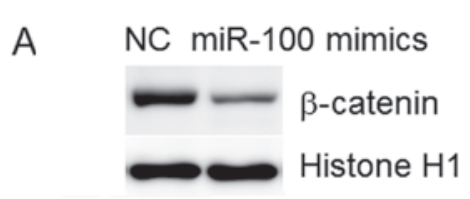

B

NC miR-100 antisense

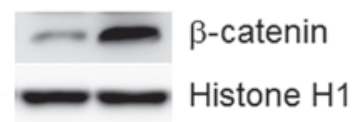

C
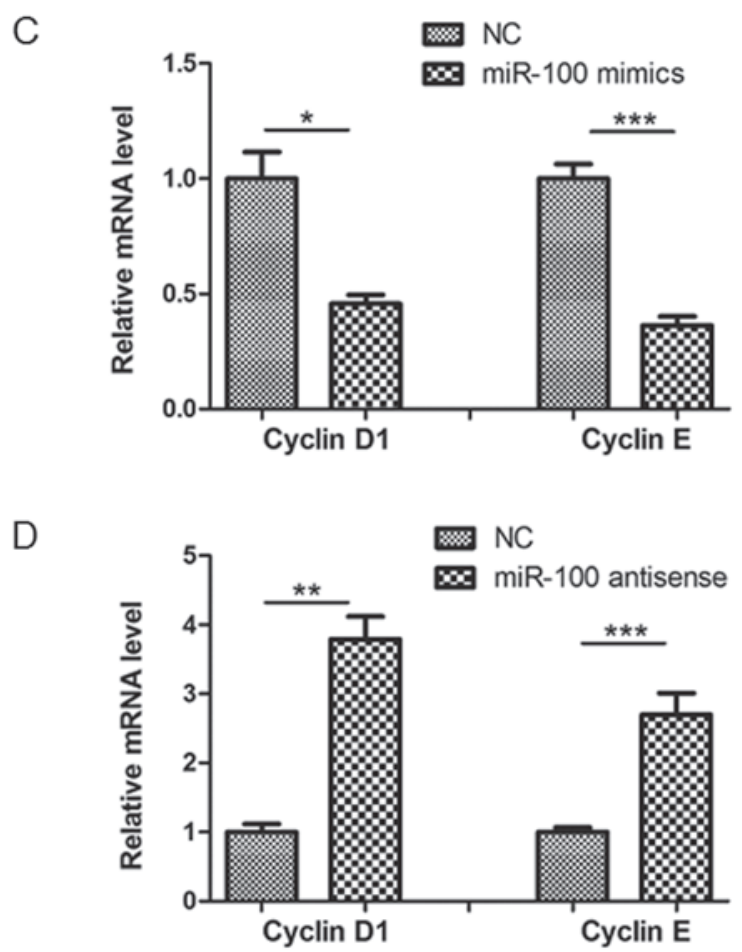

Figure 5. miR-100 regulates Wnt/ $\beta$-catenin signaling. The nuclear $\beta$-catenin expression level was determined by western blot in SW480 cells transfected with miR-100 (A) mimics or (B) antisense. Histone H1 levels were measured as a loading control. mRNA levels of cyclin D1 and E were examined by qPCR in SW480 cells transfected with miR-100 (C) mimics or (D) antisense. ${ }^{*} \mathrm{P}<0.05,{ }^{* *} \mathrm{P}<0.01$ and ${ }^{* * *} \mathrm{P}<0.001$ vs. NC. miR-100, microRNA-100; NC, negative control; qPCR, quantitative polymerase chain reaction.

enhanced by miR-100 antisense (Fig. 2A and B). Additionally, miR-100 mimics significantly inhibited the in vitro migration and invasion abilities of SW480 cells, whereas its antisense enhanced these processes (Fig. 2C and D). Similar results were also observed in the HCT116 cells (Fig. 3).

Lgr5 is a target of miR-100 in colon cancer cells. Bioinformatics software (miRWalk) was used to screen for the target gene of miR-100 in colon cancer cells. The results indicated Lgr5 to be a target of miR-100 (Fig. 4A). The luciferase activity assay established that miR-100 mimics significantly suppressed the activity of the wild-type 3'-UTR, while its antisense upregulated it. However, this effect was not observed in the mutant in SW480 cells (Fig. 4B).

Protein levels of Lgr5 and $\beta$-catenin are altered by miR-100. Consistent with the bioinformatic analysis and luciferase assay 
results, western blot analysis indicated that miR-100 mimics reduced Lgr5 protein levels compared with $\mathrm{NC}$ levels in SW480 cells, whilst miR-100 antisense produced the opposite effect (Fig. 4C and D). A previous study demonstrated that Lgr5 promotes tumor growth and progression through the activation of Wnt/ $\beta$-catenin signaling (12). In agreement with this, it was observed in the current study that miR-100 mimics inhibited, while its antisense promoted, nuclear $\beta$-catenin accumulation in SW480 cells (Fig. 5A and B). The results of the present study were in accordance with a previous study that demonstrated that cyclin D1 and E were reduced or increased by transfection of miR-100 mimics or antisense, respectively (13). This indicates that downstream targets of $\beta$-catenin are also influenced by miR-100 (Fig. 5C and D).

\section{Discussion}

Identification of cancer-specific miRNAs may be important for understanding the role they perform in tumorigenesis and exploring novel therapeutic targets. Previous studies have demonstrated that miR-100 is downregulated in several types of malignancy, including osteosarcoma, acute myeloid leukemia, and lung and hepatocellular carcinoma (14-17). miR-100 has been identified as a potential molecular marker of non-small cell lung cancer, and functions as a tumor suppressor by targeting polo-like kinase 1 (17). miR-100 has also been demonstrated to inhibit breast cancer proliferation and survival through the suppression of insulin-like growth factor 2 and $\beta$-tubulin (18). This indicates a fundamental function for miRNA as a tumor suppressor. However, the function of miR-100 in colon cancer biology remains unclear.

In the current study, the expression and potential functions of miR-100 in the regulation of the biological properties of colon cancer cells were investigated. miR-100 expression was demonstrated to be downregulated in colon cancer tissues compared with adjacent normal tissues. The subsequent gain- and loss-of-function studies suggested that miR-100 was able to reduce colon cancer cell viability, proliferation, migration and invasion in vitro.

To further explore the molecular mechanisms involved in miR-100-mediated effects on biological properties, Lgr5 was selected for further study as it was predicted to be a target of miR-100 by bioinformatics analysis. Lgr5, also known as $\mathrm{G}$ protein-coupled receptor 49, is a regulator of Wnt signaling (19). Consistent with the dysregulation of Wnt/ $\beta$-catenin signaling, Lgr5 is overexpressed in hepatocellular carcinoma, colon and ovarian cancer, basal cell carcinoma and esophageal adenocarcinoma (20-22). This suggests an important function for Lgr5 in tumorigenesis. Adenomatous polyposis coli mutations observed exclusively in Lgr5-positive cells have been identified to be able to promote adenomatous growth in the colon of mice (23). In addition to patients, the overexpression of Lgr5 has been demonstrated to correlate with poor survival of colon cancer in mice (24). However, the precise molecular mechanisms underlying the upregulation of Lgr5 in colon cancer remain to be fully elucidated, and thorough investigation is required.

Additionally, nuclear localization of $\beta$-catenin and expression of its down-stream target genes, including cyclin D1 and cyclin E were demonstrated to be regulated by miR-100. Thus, the downregulation of miR-100 may be an important mechanism for the aberrant activation of Wnt signaling in human cancer.

To the best of our knowledge, the results from the current study, for the first time explore the function of miR-100 in the progression of colon cancer. Future studies, including the generation of miR-100 knockout mice, are required to establish the physiological function of miR-100 in tumorigenesis.

\section{References}

1. Des Guetz G, Uzzan B, Bouillet T, et al: Impact of physical activity on cancer-specific and overall survival of patients with colorectal cancer. Gastroenterol Res Pract 2013: 340851, 2013.

2. Søgaard M, Thomsen RW, Bossen KS, Sørensen HT and Nørgaard M: The impact of comorbidity on cancer survival: a review. Clin Epidemiol 5 (Suppl 1): 3-29, 2013.

3. Chiu BC, Ji BT, Dai Q, Gridley G, McLaughlin JK, Gao YT, Fraumeni JF Jr and Chow WH: Dietary factors and risk of colon cancer in Shanghai, China. Cancer Epidemiol Biomarkers Prev 12: 201-208, 2003.

4. Walker AS, Zwintscher NP, Johnson EK, Maykel JA, Stojadinovic A, Nissan A, Avital I, Brücher BL and Steele SR: Future directions for monitoring treatment response in colorectal cancer. J Cancer 5: 44-57, 2014.

5. Sun K and Lai EC: Adult-specific functions of animal microRNAs. Nat Rev Genet 14: 535-548, 2013.

6. Ameres SL and Zamore PD: Diversifying microRNA sequence and function. Nat Rev Mol Cell Biol 14: 475-488, 2013.

7. Wu WK, Law PT, Lee CW, Cho CH, Fan D, Wu K, Yu J and Sung JJ: MicroRNA in colorectal cancer: from benchtop to bedside. Carcinogenesis 32: 247-253, 2011.

8. Kjaer-Frifeldt S, Hansen TF, Nielsen BS, Joergensen S, Lindebjerg J, Soerensen FB, dePont Christensen R and Jakobsen A: The prognostic importance of miR-21 in stage II colon cancer: a population-based study. Br J Cancer 107: 1169-1174, 2012.

9. Wu W, Yang J, Feng X, Wang H, Ye S, Yang P, Tan W, Wei G and Zhou Y: MicroRNA-32 (miR-32) regulates phosphatase and tensin homologue (PTEN) expression and promotes growth, migration, and invasion in colorectal carcinoma cells. Mol Cancer 12: 30, 2013.

10. Yuan K, Xie K, Fox J, Zeng H, Gao H, Huang C and Wu M: Decreased levels of miR-224 and the passenger strand of miR-221 increase MBD2, suppressing maspin and promoting colorectal tumor growth and metastasis in mice. Gastroenterology 145: 853-864, 2013.

11. Schetter AJ, Leung SY, Sohn JJ, Zanetti KA et al: MicroRNA expression profiles associated with prognosis and therapeutic outcome in colon adenocarcinoma. JAMA 299: 425-436, 2008.

12. Haegebarth A and Clevers $\mathrm{H}$ : Wnt signaling, lgr5, and stem cells in the intestine and skin. Am J Pathol 174: 715-721, 2009.

13. Wilson C: Diabetes: Human $\beta$-cell proliferation by promoting Wnt signalling. Nat Rev Endocrinol 9: 502, 2013.

14. Huang J, Gao K, Lin J and Wang Q: MicroRNA-100 inhibits osteosarcoma cell proliferation by targeting Cyr61. Tumour Biol 35: 1095-1100, 2014.

15. Zheng YS, Zhang H, Zhang XJ, Feng DD, et al: MiR-100 regulates cell differentiation and survival by targeting RBSP3, a phosphatase-like tumor suppressor in acute myeloid leukemia. Oncogene 31: 80-92, 2012.

16. Chen P, Zhao X and Ma L: Downregulation of microRNA-100 correlates with tumor progression and poor prognosis in hepatocellular carcinoma. Mol Cell Biochem 383: 49-58, 2013.

17. Liu J,Lu KH,Liu ZL, Sun M, De W and Wang ZX: MicroRNA-100 is a potential molecular marker of non-small cell lung cancer and functions as a tumor suppressor by targeting polo-like kinase 1 . BMC Cancer 12: 519, 2012.

18. Gebeshuber CA and Martinez J: miR-100 suppresses IGF2 and inhibits breast tumorigenesis by interfering with proliferation and survival signaling. Oncogene 32: 3306-3310, 2013.

19. Leushacke M and Barker N: Lgr5 and Lgr6 as markers to study adult stem cell roles in self-renewal and cancer. Oncogene 31: 3009-3022, 2012. 
20. Fukuma M, Tanese K, Effendi K, Yamazaki K, Masugi Y, Suda M and Sakamoto M: Leucine-rich repeat-containing G protein-coupled receptor 5 regulates epithelial cell phenotype and survival of hepatocellular carcinoma cells. Exp Cell Res 319: 113-121, 2013.

21. Zeki SS, Graham TA and Wright NA: Stem cells and their implications for colorectal cancer. Nat Rev Gastroenterol Hepatol 8 : 90-100, 2011.

22. Schuijers J and Clevers H: Adult mammalian stem cells: the role of Wnt, Lgr5 and R-spondins. EMBO J 31: 2685-2696, 2012.
23. Lewis A, Segditsas S, Deheragoda M, et al: Severe polyposis in Apc(1322T) mice is associated with submaximal Wnt signalling and increased expression of the stem cell marker Lgr5. Gut 59: 1680-1686, 2010.

24. Liu Z, Dai W, Jiang L and Cheng Y: Over-expression of LGR5 correlates with poor survival of colon cancer in mice as well as in patients. Neoplasma 61: 177-185, 2014. 